\title{
La terminologie comparée au service de l'enseignement en langue de spécialité en 3e cycle DEA Monnaie-Finance-Banque
}

\section{Catherine Resche}

\section{(2) OpenEdition \\ Journals \\ Édition électronique \\ URL : http://journals.openedition.org/asp/3501 \\ DOI : 10.4000/asp.3501 \\ ISSN : 2108-6354 \\ Éditeur \\ Groupe d'étude et de recherche en anglais de spécialité}

\section{Édition imprimée}

Date de publication : 1 décembre 1996

Pagination : 195-205

ISSN : 1246-8185

\section{Référence électronique}

Catherine Resche, «La terminologie comparée au service de l'enseignement en langue de spécialité en 3e cycle DEA Monnaie-Finance-Banque », ASp [En ligne], 11-14 | 1996, mis en ligne le 19 octobre 2013, consulté le 23 avril 2019. URL : http://journals.openedition.org/asp/3501 ; DOI : 10.4000/ asp.3501

Ce document a été généré automatiquement le 23 avril 2019.

Tous droits réservés 


\title{
La terminologie comparée au service de l'enseignement en langue de spécialité en 3 e cycle DEA Monnaie-Finance- Banque
}

\author{
Catherine Resche
}

\section{NOTE DE L'ÉDITEUR}

L'auteur de cette contribution n'a pas autorisé sa publication en ligne.

\section{RÉSUMÉS}

Depuis trois ans, avec un public d'étudiants de $3^{\mathrm{e}}$ cycle en DEA de Monnaie-Finance-Banque, je m'efforce de construire un pont entre l'enseignement de l'anglais de spécialité et les autres cours de ce DEA économique. Après une introduction aux principes de méthodologie en terminologie comparée, je fais travailler les étudiants à partir d'un corpus de textes techniques authentiques, puis je leur demande de rédiger pour la fin de l'année un mini-mémoire de 30 couples de fiches de terminologie sur le domaine choisi pour leur mémoire général de DEA. Par ce biais, l'identification et la reconnaissance des termes nouveaux deviennent plus méthodiques et leur réutilisation plus spontanée. La compréhension de l'écrit et de l'oral sont facilitées et l'on remarque que l'expression écrite et orale, aussi bien que la traduction, gagnent en qualité. 
This paper deals with my three-year experience of teaching post-graduate students specializing in "Money, Finance and Banking". I have tried to create a link between their seminars and lectures in economics and my English course. After introducing the methodological principles of comparative terminology, I have the students work on concrete examples selected from a corpus of genuine, technical texts. I also ask them to do some research work into a field of their choice which is to result in a 60-page terminological paper - on both English and French terms - by the end of the year. I have found that this focus on comparative terminology helps them to read reference books more efficiently, to identify and memorize new terms and use them more spontaneously. Their ability to understand written as well as spoken financial English is greater and their communication skills in ESP improve in the process; they also become better at translating technical texts.

\section{INDEX}

Keywords : comparative terminology, contextual analysis, oral expression, phraseology, research paper in terminology, terminological unit, translation, written expression

Mots-clés : analyse de contextes, dossier terminologique, expression écrite, expression orale, phraséologie, terminologie comparée, traduction, unité terminologique

\section{AUTEUR}

\section{CATHERINE RESCHE}

Agrégée d'anglais, Catherine Resche a ensuite choisi de faire un DEA de linguistique avant d'entreprendre une thèse de terminologie comparée sur la Bourse des Valeurs, qu'elle a soutenue en 1985. Elle est maintenant maître de conférences à l'Université de Paris 2 Panthéon-Assas où elle a la charge, entre autres responsabilités, d'enseigner l'anglais en DEA Monnaie-FinanceBanque. Elle y a également créé, en collaboration avec un autre maître de conférences, le diplôme du Certificat d'anglais pour économistes (CAPEC), qui se prépare en deux ans (niveaux licencemaîtrise) à raison de trois heures hebdomadaires. Elle s'intéresse à tous les aspects de la langue de spécialité : terminologie, phraséologie, dictons, métaphores, néologie, normalisation et aux problèmes de la traduction. catherineresche@club-internet.fr 